\title{
Measuring How the Degree of Content Knowledge Determines Performance Outcomes in an Engineering Design-Based Simulation Environment for Middle School Students
}

\begin{abstract}
Many secondary schools offer STEM-based technology and engineering courses that use simulation modeling to teach engineering design. However, the performance of the final design is usually dependent upon the student's ability for knowledge application. When a student reaches the limit of this content knowledge, they may resort to other problem solving processes, such as a trial and error approach, to improve their design. This study measures the outcomes of students with varying levels of content knowledge to determine how the level of knowledge determines their performance within a virtual design simulation. The results show that students with a greater content knowledge initially have significantly better performance outcomes. However, if given enough opportunities to engage in the simulation activity, students with less content knowledge perform equally well.
\end{abstract}

Keywords: secondary education; simulations; improving classroom teaching; interactive learning environments; pedagogical issues

\subsection{Introduction}

During a traditional technology education-based learning process, students are typically first exposed to the content by the classroom teacher and then given the opportunity to apply their knowledge to solve a problem. In technology education classrooms with an engineering context, students usually apply a problem solving process by developing a project or artifact to demonstrate their understanding of the material (Mentzer, 2011). As part of this process students are expected to apply the knowledge they have learned to make decisions that lead to a solution for the problem. Technology and engineering education students may have the opportunity to use a variety of tools, including simulation modeling, to solve an engineering design problem. Computer simulations are common in an engineering-based curriculum. However, it is important for classroom teachers to understand how the integration of computer simulations with traditional instruction affects the performance outcomes of the learning objectives. This research describes how different levels of knowledge application affect the performance of students when using a computer simulation modeling program as part of an engineering design project.

\subsection{Simulation Modeling}

Simulation modeling, virtual modeling, solid modeling, and predictive analysis are tools we as humans have available in order to extend human capabilities. Computer simulation was defined by de Jong and van Joolingen (1998) as "a program that contains a model of a system (natural or artificial) or a process". Computer-aided three-dimensional design is a technological extension to traditional hands-on drafting and rendering. Three-dimensional computer aided design can also teach students advanced mathematical and scientific concepts, through a variety of different software programs. Simple machines, mechanical advantage, related mathematics, 
and problem solving are all examples of integration of mathematics and science into virtual learning software (Smith, 2003). Blanchard et al (2010) showed inquiry-based science laboratory activities increased gains in student achievement and long-term retention compared to students that learned from traditional laboratory instruction. When integrating similar concepts into the design process, it has been documented that gains in learning outcomes may also occur within certain simulation-related learning activities (Jacobson, Taylor, Richards, \& Lai, 2013). By using three-dimensional computer-aided design, analysis, and simulation software, students are able to create virtual representations of physical objects. Along with three-dimensional software programs, simulation software can be used to analyze a design. Simulations allow students to see how various functions are interrelated and help contribute to performance outcomes (Lamoureux, 2009). Virtual learning environments allow varying interactions and encounters with the participant in order to provide a wide range of learning capabilities (Piccoli, Ahmad, \& Ives, 2001). Students are able to use these programs to experiment with different scenarios, problem solving, and decision making with little risk and without wasting resources.

Simulations allow users to examine resulting values of computations after establishing the parameters of the system (Smith \& Pollard, 1986). The parameters can then be adjusted, based on the results, and further computations can be analyzed. Simulation has traditionally been used as a tool to apply the content knowledge learned. Once the theoretical knowledge is learned, simulation modeling is introduced as a tool to demonstrate the application of the learned theory. By using simulations, students can test a greater number of models and run multiple iterations of testing before committing to a final solution. This provides an efficient method of knowledge application because students can learn how the theory is used in multiple situations and can gain knowledge about the authentic application of course content. Engineering concepts can be introduced to the students, and then simulation modeling can be integrated in order for students to apply the learned knowledge. Simulation modeling can be extremely beneficial in expanding student learning when used in combination with physical models to illustrate engineering and design concepts (Clark \& Ernst, 2006; Ernst \& Clark, 2009; Jaakkola, Nurmi, \& Veermans, 2011; Newhagen, 1996; Smith \& Pollard, 1986; Zacharia, 2007). By incorporating simulation modeling into the lesson, students can create multiple virtual models, as wells as test and redesign them as necessary (Deal III, 2002; Piccoli, Ahmad \& Ives, 2001).

\subsection{Knowledge Application}

Virtual model simulations can be particularly useful in a project-based environment commonly found in technology education curriculum. For example, bridge building and $\mathrm{CO} 2$ cars are two popular middle grade activities. However, both of these activities require consumable materials and substantial time to complete physical models. It would be extremely difficult for a classroom teacher to spend the time necessary for students to participate in the testing, evaluation, and redesign steps of the engineering design process using only physical models as the artifact. Students commonly use virtual modeling to apply their knowledge to all the steps of the engineering design process and complete the learning loop for testing and redesign.

Ideally, students will apply the content knowledge gained through classroom instructions to achieve the desired simulation performance outcomes. However, in this type of environment, with multiple iterations of design, content knowledge may reach its limit and more of a trial and error approach may enter the learning process. In a design-based learning environment, content 
knowledge gained by the students contains a variety of aspects used during the problem solving process. As students are gaining knowledge through content delivery, these would include but are not limited to; theoretical knowledge, procedural knowledge, knowledge of variables and constraints, and knowledge application. This would also include knowledge of the process that cannot be directly linked to a learned concept. This is when a trial and error approach may be embedded into the overall design process.

The concept of trial and error has historically been used as one method of problem solving. When learning through trial and error, the learner is required to choose among many alternative outcomes. (Noble, 1957). This method is typically unsystematic and does not follow any sort of methodology, therefore a trial and error approach begins once the student has reached the limit of theoretical knowledge (Callander, 2011). A student using this method may have little knowledge in the problem area, and when the problem reaches a certain level of difficulty, there is a possibility of unknown conditions. When little is known, theory often provides limited guidance (Callander, 2011).

Although a specific methodology is not uniformly used, trial and error processes are rarely random (Hull, 1939). Learning occurs through information gained from experience, and a concept of knowledge is built upon this learning process. When a student rejects choices that are erroneous, meaning they will not benefit from the situation, they can learn by trial and error (Young, 2009). The approach is not careless, but can be organized and logical. Students that engage in computer simulation activities before gaining significant content knowledge, have been shown to demonstrate high levels of academic performance in computational model-based learning environments (Bowen \& DeLuca, 2015; Jacobson, Kim, Pathak \& Zhang, 2013). Kapur (2008) reported that allowing students to struggle longer through learning activities above their ability level may create longer retention of learning gains. Students are able to construct frameworks of knowledge that enable them to solve problems without consciously applying a specific concept. After multiple iterations of the process, the learner may sort through a variety of possible scenarios in order to manipulate the variables. Callander (2011) states, "The search for good outcomes is frequently guided by trial and error." Students will experiment with alternative outcomes, maintaining the latest approach only if it provides an advantage. The seeking out of new strategies and ideas allows this method to help a student find new knowledge (Young, 2009).

The continuous process of using a trial and error method of problem solving is seldom random as participants learn from their mistakes and experiences (Jones, 1945). Each time a choice is made, and a student tries a new tactic, valuable content is exposed. A learner will use accumulated information, from both successes and failures, to direct all other future selections (Callander, 2011). As learning occurs through the trial and error process, students will show a strengthening of the correct tendencies and a weakening of the incorrect tendencies (Hull, 1939). The objective, when using trial and error learning, is to offer insight on when and how learners experiment with their decisions and if they are able to sufficiently learn about the topic to achieve effective outcomes (Callander, 2011).

\subsection{Research Question}

Although there is research about simulation modeling, there is little research as to how much of the content knowledge gained by the student is being applied and aligned to the performance outcome of the engineering design process. When using a simulation platform, 
students are generally expected to apply the knowledge gained through the learned content to the simulation portion of the virtual environment. However, at some point, students may exhaust their content knowledge and may then resort to more of a trial and error approach in an attempt to improve the design of their virtual models. As long as the student feels they have adequate knowledge to improve a design, they will apply content knowledge until the maximum level of knowledge application is reached. Current research about knowledge application focuses mostly on adult usage, such as post-secondary students and practicing professionals. There is extensive research on using computer simulations in regards to solving scientific and engineering-based problems; however, there is little research on this topic in the context of classroom learning in technology education at the secondary level. The researchers of the current study have designed the methodology of this research project to determine how much content knowledge is a factor in achieving performance outcomes in a virtual design simulation environment for middle school students.

The current project is designed to measure the extent students are using content knowledge and trial and error to solve an engineering problem. As mentioned in section 1.2, trial and error may be one of the components integrated into the procedural knowledge throughout the application of the design process. The intent of this study is not to determine the extent to which content knowledge application and trial and error are integrated throughout the design process; rather the purpose of this study is to determine if there is a difference in the performance outcomes when the content knowledge is controlled between two groups of participants. The context of the engineering problem in this study is the design of a bridge through the use of a virtual bridge design simulation program. This study is designed to answer the following question: How does the level of content knowledge affect the performance outcomes of designed-based virtual bridge models in a computer simulation environment?

\subsection{Methodology}

The purpose of this research project is to measure if a significant difference exists in various aspects of integrating content knowledge and virtual bridge design resulting in different levels of knowledge application. In this project the performance outcome was measured by the efficiency of the students' virtual bridge models. Students in two separate groups, control and experimental, were exposed to different levels of activities throughout the simulation program, therefore resulting in different levels of content knowledge. Then, by allowing all the students to design virtual models, the researchers measured how different levels of content knowledge affected the student's ability to apply the learned knowledge. The following sections describe the methodology of the research project, how the content level for students was determined, and how the simulation program was used to collect data.

\subsection{Structures Virtual Platform Description}

For the secondary classroom, many computer simulation packages exist for designing virtual models of bridges. For this research project, the software application Structures 2.0, published by Whitebox Learning, was used to measure student performance. Whitebox Learning is a STEM-focused virtual modeling platform with several different web-based applications.

Structures 2.0 is one of the applications in the software package, and was the application used in this study. There are two versions of the Structures 2.0 application available for the secondary 
classroom; one for the middle school level and one for the high school level. The difference in the two versions is the amount of mathematics required to complete the research section and formative assessments built into the program. The high school version uses more advanced mathematics to solve for the different types of forces in each of the bridge truss members. The middle school version uses less mathematical concepts and focuses on overall conceptual knowledge of bridge efficiency. This study used the middle-school version of the Structures 2.0 application.

The Structures 2.0 platform integrates several different aspects of STEM learning. Since this is a web-based platform, students can access the program from wherever they have internet access by logging in with a username and password unique to each student. After the student logs in, the program begins with an introduction section providing background knowledge about general bridge design principles. This section provides basic engineering concepts such as truss components, factors of safety, forces, and other definitions related to general bridge design. The next section of the program, research, provides an in-depth knowledge of truss design and bridge efficiency. Throughout the research section, formative assessments are built in to measure the students' understanding of the content covered in the research section. The teacher can monitor the students' progress in the teacher control center. Once the research section is complete, the students follow a virtual tutorial demonstrating the use of the specific program functions needed to design a truss. Once the tutorial section is completed, the engineering section allows students to design their own bridges. As the students work through different designs, they can test them to see how much weight the truss can support before failure. Each test is recorded as an iteration, and based on the specifications predetermined by the teacher, these iterations can be within specifications or out of specifications. The program only records bridge efficiencies for in-spec iterations. Once the student has decided on a final design, a template of the truss can be printed for building the physical model. The students build two identical trusses that are joined together to make a bridge which can be tested for efficiency. Please refer (Bowen \& DeLuca, 2015) for a more detailed description of the Whitebox Learning Structures 2.0 platform.

\subsection{Research Participants}

The participants in this research project were from a middle school located in the upper midwest of the United States. All students at this school are required to register for nine-weeks, or one quarter, of a STEM-based technology education course. The students in this study were 8th grade students of one of the technology education teachers. Due to the course lasting one quarter, the classroom teacher has a new student roster each quarter. The research project involved four classes throughout the day and was administered over two quarters during the 2013-2014 school year. A control group and experimental group were formed, with two classes each quarter being in each group. These classes were of mixed ability with no intentional grouping of the students by the school. However, due to measures beyond the researchers' control that determine student scheduling, this study is categorized as quasi-experimental and assumes non-parametric conditions. Each quarter, the classes were randomly selected to be in the control and experimental groups. 


\subsection{Control and Experimental Group}

After the researchers obtained student and parental consent at the beginning of each quarter, both the control and experimental groups took a pre-test before beginning the Structures 2.0 program. After the pre-test, all the students in both groups were given login information for the web-based platform. The difference between the control and experimental groups was whether or not the students were exposed to the introduction and research sections of the Structures 2.0 platform, which provides the theoretical knowledge of bridge design. The control group proceeded through all the sections of the program as they are designed; introduction, research, virtual tutorial, and engineering. Therefore, the control group is gaining more content knowledge when compared to the experimental group by initially engaging in the introduction and research sections. Also, for the control group, the tutorial for the bridge design process is embedded within the program. The experimental group began the program by skipping the introduction and research sections and proceeding directly to the engineering section. Each student in the experimental group was given a paper copy of the tutorial. Due to some of the content knowledge being embedded within the virtual tutorial, a paper copy was provided to the experimental group allowing these students to learn how to use the program functions for designing their bridges without the risk of being exposed to any of the structures content knowledge. The introduction and research sections were providing content knowledge to the control group whereas the tutorials, both virtual and paper copies, were providing the functional knowledge of the engineering section to both groups. Once both groups finished the virtual models in the engineering section, the truss templates were printed and the physical models were constructed. Once all of the physical models were tested, the students in both groups took the post-test. A summary of the sequencing of activities for each group is shown in Fig. 1.

Control: $\quad$ Pre-test $>$ Introduction $>$ Research $>$ Virtual Tutorial $>$ Engineering Design and Analysis $>$ Physical Model > Post-test

Experimental: Pre-test > Paper Copy of Tutorial > Engineering Design and Analysis > Physical Model $>$ Post-test

Figure 1. Sequencing for Control and Experimental Groups

\subsection{Data Collection and Analysis}

While the students are navigating through the structures application, the researchers can monitor the students' activities through the teacher control center. The teacher control center captures different aspects of the students' progress. In this research study, the virtual simulation data captured from the teacher control center is shown below. 


\begin{tabular}{|l|l|}
\hline Item & Measured \\
\hline Efficiency of the first virtual model & Total weight held divided by weight of the bridge \\
\hline Efficiency of the best virtual model & Total weight held divided by weight of the bridge \\
\hline In-Spec Iterations & \# tests for each design within specifications \\
\hline Out-of-Spec Iterations & \# tests for each design out of specifications \\
\hline Total Iterations & Total \# of in-spec and out-of-spec iterations combined \\
\hline
\end{tabular}

The program also captures the students' progress through the formative assessments, the average grade of the formative assessments, and the time on task spent throughout the research portion of the program. However, since the experimental group did not participate in these aspects of the program, this data is not included in the study. In addition to the virtual data, the efficiency of the physical model was also collected to measure engineered performance.

The traditional pre- and post-tests used in the study were administered to measure student achievement of content knowledge. The pre- and post-tests consisted of 15 multiple choice items to measure the students' understanding of the content represented in the Structures 2.0 platform. The items for these tests were developed by the researchers based on the content covered through the research portion of the Structures 2.0 application and reviewed by experts to ensure content validity. The teacher used the formative assessments and the post-test as part of the student's grade in order to improve the possibility of students giving maximum effort during the assessments.

After the data was collected, a statistical analysis was conducted to measure any significant differences for various factors between the groups. Due to the non-parametric conditions of the study, the Wilcoxon Scores (Rank Sums) two-sample test was used for the statistical analysis.

\subsection{Results}

The results of the statistical analysis for the pre- and post-tests are shown in Table 1. 
Table 1.

Statistical analysis of pre- and post-assessments

\begin{tabular}{|c|c|c|c|c|c|c|}
\hline Item & $\mathrm{N}$ & Min & Max & $\begin{array}{l}\text { Sum of } \\
\text { Ranks }\end{array}$ & $\mathrm{Z}$ & P-value \\
\hline \multicolumn{7}{|l|}{ Pre-test } \\
\hline Control & 64 & 4 & 12 & 4246 & -0.19 & 0.850 \\
\hline Experimental & 69 & 3 & 13 & 4665 & & \\
\hline \multicolumn{7}{|l|}{ Post-test } \\
\hline Control & 63 & 5 & 14 & 4434 & 1.61 & 0.107 \\
\hline Experimental & 66 & 3 & 14 & 3951 & & \\
\hline \multicolumn{7}{|c|}{ Difference (Post minus Pre) } \\
\hline Control & 63 & -3 & 6 & 4528 & 2.05 & $0.040 *$ \\
\hline Experimental & 66 & -5 & 7 & 3858 & & \\
\hline
\end{tabular}

From these results, the control group had a significantly higher difference in the change in tests scores. There was not a significantly difference between the two groups when comparing the pre-test scores and the post-test scores separately.

Table 2.

Statistical analysis for virtual and physical model efficiencies

\begin{tabular}{|c|c|c|c|c|c|c|}
\hline Item & $\mathrm{N}$ & Min & Max & $\begin{array}{c}\text { Sum of } \\
\text { Ranks }\end{array}$ & $\mathrm{Z}$ & P-value \\
\hline \multicolumn{7}{|c|}{ First virtual model efficiency } \\
\hline Control & 59 & 1020 & 4830 & 4148 & 1.97 & $0.049 *$ \\
\hline Experimental & 67 & 627 & 4880 & 3854 & & \\
\hline \multicolumn{7}{|c|}{ Best virtual model efficiency } \\
\hline Control & 59 & 1020 & 5270 & 3725 & -0.11 & 0.916 \\
\hline Experimental & 67 & 1380 & 5280 & 4277 & & \\
\hline \multicolumn{7}{|c|}{ Difference in Best and First virtual efficiencies } \\
\hline Control & 61 & 0 & 4080 & 3662 & -1.44 & 0.149 \\
\hline Experimental & 68 & 0 & 4350 & 4723 & & \\
\hline
\end{tabular}

Difference in Best and First virtual efficiencies; w/o students with only one valid item
Control
50
$0 \quad 4080$
2741
$-0.63$
0.529
Experimental
63
4350
3700

Physical model efficiency

\begin{tabular}{lllllll} 
Control & 64 & 48 & 1534 & 4379 & 1.03 & 0.303 \\
Experimental & 65 & 98 & 1336 & 4006 & & \\
\hline
\end{tabular}

*significant at $\alpha=.05$ 
Table 3.

Statistical analysis for virtual model iterations

\begin{tabular}{|c|c|c|c|c|c|c|}
\hline Item & $\mathrm{N}$ & Min & Max & $\begin{array}{c}\text { Sum of } \\
\text { Ranks }\end{array}$ & $\mathrm{Z}$ & P-value \\
\hline \multicolumn{7}{|l|}{ In-spec iterations } \\
\hline Control & 64 & 0 & 39 & 3741 & -2.47 & $0.014 *$ \\
\hline Experimental & 69 & 0 & 55 & 5171 & & \\
\hline \multicolumn{7}{|c|}{ Out-of-spec iterations } \\
\hline Control & 64 & 0 & 54 & 3656 & -2.58 & $0.004 *$ \\
\hline Experimental & 69 & 0 & 70 & 5256 & & \\
\hline \multicolumn{7}{|l|}{ Total iterations } \\
\hline Control & 64 & 1 & 68 & 3598 & -3.11 & $0.002 *$ \\
\hline Experimental & 69 & 1 & 82 & 5314 & & \\
\hline
\end{tabular}

The results for the statistical analysis for the model efficiencies and the number of simulation iterations are shown in tables 2 and 3, respectively. From these results, the control group had a significantly higher efficiency for the first virtual model design. There was not a significant difference in the best virtual model efficiencies. The difference between the best and first efficiency for each student was analyzed to determine if there was a significant difference in the overall gain in efficiency between the two groups. This measure was analyzed a second time not including the students with only one iteration. Since the program only records efficiencies for in-spec iterations, having only one in-spec iteration would automatically results in an efficiency gain of zero. The researchers also felt that having only one iteration does not allow the student to properly demonstrate an iterative design process format. However, for both of these tests, there was not a significant difference between the two groups. There was also not a significant difference for the physical model efficiency.

In regards to the number of iterations, the experimental group had a significantly higher number of in-spec, out-of-spec, and total iterations. Although the time spent on each of the program's sections was not included in the data for this research design, the experimental group skipped the research section and proceeded directly to engineering section of the program. Therefore, the experimental group had more time to design virtual models, resulting in significantly more iterations than the control group.

\subsection{Discussion}

The purpose of this analysis was to approach the research question from different aspects in order to draw a conclusion as to how the students' demonstrated different levels of knowledge and performance from exposure to various levels of content within a simulation environemnt. The results show there is a significant difference in the means of the difference between the preand post-tests between the two groups. This is expected since the control group was exposed to the content through the introduction and research sections of the virtual platform while the 
students in the experimental group were not. The results also show there is a significant difference in the means of the first virtual bridge design efficiency between the control and experimental groups. This demonstrates that once the students gain initial content knowledge about truss design, this knowledge is being applied during the initial design of their bridge.

However, there is not a significance difference in the means of the best virtual model efficiency between the two groups. This suggests there are other factors contributing to the students thought process during a simulation activity. It is possible that content knowledge is a significant factor when first designing a truss, but at some moment within the activity, other procedural knowledge is being applied by both groups resulting in no significant difference in the best efficiency. The students in the experimental group were not exposed to the content in the introduction and research sections of the program. Therefore, since content knowledge was not part of the instructional process, these students had to rely on different aspects of procedural knowledge to increase their design efficiency when negotiating through multiple iterations. Since they went directly to the engineering section, these students also had more time to spend on the virtual design and simulation portion of the program, resulting in a significantly higher number of iterations. In regards to the engineering design process, these students essentially spent their entire simulation project time in the design, test, and redesign phases. Because of the significantly higher number of iterations, these students were able to demonstrate knowledge application in the context of the specific program functions. Therefore, over a longer period of time, they were able to gain enough application and procedural knowledge to design bridges that were not significantly less efficient than the control group.

These results can have a significant impact on how instructors choose to assess a student's performance. These results suggest a student, when given enough iterations, can achieve performance outcomes that are not significantly different with less content knowledge than students with a greater amount of initial content knowledge. This could have substantial influence on how classroom instruction is designed when integrating simulation modeling with traditional instruction. As this study demonstrates, exposing students to more content knowledge produces significantly higher outcomes on traditional assessments as shown by the difference in pre- and post-test scores. In regards to performance application, this initial content knowledge produces significantly greater initial bridge efficiencies. However, given enough opportunities, students with less content knowledge can demonstrate a performance outcome that is not significantly different than students with more content knowledge. Therefore using performance outcomes as the only measure of student knowledge would not be an accurate assessment of their content knowledge.

\subsection{Conclusion}

Through the methodology included in this research project, the researchers have concluded that content knowledge plays a significant factor in the initial knowledge application phase of the simulation performance environment. However, students with less content knowledge can design virtual model that are not significantly different if given enough iterations. From the design of this study, it could not be determined at what point the students in the experimental group achieve a virtual design that is not significantly different. This is an area for future research. Additional research is also needed to determine to what degree students are integrating content knowledge, procedure knowledge, experimental knowledge, and trial and error to achieve these outcomes. 


\subsection{Ad Hoc Study}

In response to the data collected in this research, an ad hoc study was conducted to gather additional data about how students are applying content knowledge along with procedural knowledge in a simulation environment. The same Whitebox Learning Structures 2.0 platform was used by a group of 10 students at a university in the southeastern United States. These students were in a class as part of the Technology, Engineering, and Design department in the School of Education. The assignment used the high school version of the Structures 2.0 program to create the most efficient bridge possible within two class periods, a total work time of approximately 4 hours. At the conclusion of the assignment, the students participated in a brief interview to collect data on how they used various aspects of the engineering design process within the context of the simulation program. The researchers were attempting to capture the level of knowledge application each student believed was used during the process. The sequence of activities in this study followed the same design as the control group in the previous study. The results of the interview are shown in Table 4.

Table 4.

Results of interview questions for ad hoc study

\begin{tabular}{lcccc} 
Item & & Yes & & No \\
\cline { 1 - 3 } Did you have previous knowledge of bridge design & & 5 & & 5 \\
Was the virtual simulation beneficial in learning about bridge design & & 10 & & 0 \\
Did you use a trial and error process at any point during the simulation & & 5 & 5 \\
Was your most efficient design the last file saved & & 6 & 4 \\
\hline
\end{tabular}

These results show that at some point during the simulation design process, half of the students reported using a trial and error approach. It also shows that out of the 10 students, 4 of them reported that their most efficient design was not the last design they created. This shows that at some point, improvements are not being made and a previous design may be the best one.

The researchers believe that at some point, theoretical knowledge reaches a limit within the knowledge application process, and therefore, over time, may not be significantly different than being exposed to more knowledge application itself. According to the design of this study, at some point during the knowledge application process in a simulation environment, theoretical knowledge reaches a limit and trial and error becomes a part of the design process. The ad hoc study, although brief, supports the findings of the current study by documenting student responses indicating that trial and error was part of their design process. These conclusions are important when determining the objective of the project activity and the balance of content knowledge versus knowledge application within a curriculum design.

\subsection{Future Research}

This research study demonstrates the need to understand how students are applying different aspects of content and procedural knowledge throughout engineering-based simulation activities. Further research is needed to determine at what point and to what extent in knowledge 
application a student integrates different aspects of procedural knowledge to improve their virtual designs. The results of this study led the researchers to develop a brief survey to be administered to the middle school students at the conclusion of the simulation design project in future semesters. This survey, along with other data collection methods, will be refined to better capture how students are integrating content knowledge with knowledge application when producing virtual designs.

\section{References}

Blanchard, M., Southerland, S., Osborne, J., Sampson, V., Annetta, L., \& Granger, E. (2010). Is inquiry possible in light of accountability?: A quantitative comparison of the relative effectiveness of guided inquiry and verification laboratory instruction. Science Education, 94(4), 577-616.

Bowen, B. D. \& DeLuca, V. W. (2015). Comparing traditional versus alternative sequencing of instruction when using simulation modeling. Journal of STEM Education: Innovations and Research, 16(1), 5-10.

Callander, S. (2011). Searching and learning by trial and error. The American Economic Review, 101(6), 2277-2308.

Clark, A.C. \& Ernst, J.V. (2006). Supporting technology literacy through the integration of engineering, mathematic, scientific, and technological concepts. Published Proceedings of the American Society for Engineering Education Annual Conference and Exposition, Chicago, IL.

Deal III, W. F. (2002). Distance learning: teaching technology online. Technology Teacher, 61(6), 21-27.

de Jong, T. \& van Joolingen, W. R. (1998). Scientific discovery learning with computer simulations of conceptual domains. Review of Educational Research, 68(2), 179-201.

Ernst, J. V. \& Clark, A. C. (2009). Technology-based content through virtual and physical modeling: a national research study. Journal of Technology Education, 20(2), 23-36.

Hull, C. L. (1939). Simple trial and error learning--an empirical investigation. Journal of Comparative Psychology, 27(2), 233-258.

Jaakkola, T., Nurmi, S. \& Veermans, K. (2011). A comparison of students' conceptual understanding of electric circuits in simulation only and simulation-laboratory contexts. Journal of Research in Science Teaching, (48)1, 71-93.

Jacobson, M., Kim, B., Pathak, S., \& Zhang, B. (2013). To guide or not to guide: issues in the sequencing of pedagogical structure in computational model-based learning. Interactive Learning Environments. DOI: 10.1080/10494820.2013.792845

Jacobson, M., Taylor, C., Richards, D., \& Lai, P. (2013). Computational Scientific Inquiry With Virtual Worlds and Agent-Based Models: New Ways of "Doing" Science to Learn Science. Presentation at the annual meeting of the American Educational Research Association. San Francisco, CA.

Jones, H. E. (1945). Trial and error learning with differential cues. Journal of Experimental Psychology, 35(1), 31-45.

Kapur, M. (2008). Productive failure. Cognition and Instruction, 26(3), 379-424. 
Lamoureux, K. (2009, October 10). Experiential leadership. Leadership Excellence.

Mentzer, N. (2011). High School Engineering and Technology Education Integration through Design Challenges. Journal of STEM Teacher Education, 48(2), 103-136.

Newhagen, J. E. (1996). Why communication researchers should study the internet: a dialogue. Journal of Communication, 46(1), 4-13.

Noble, C. E. (1957). Human trial-and-error learning. Psychological Reports, 3, 377-398.

Piccoli, G., Ahmad, R., \& Ives, B. (2001). Web-based virtual learning environments: A research framework and a preliminary assessment of effectiveness in basic it skills training. MIS Quarterly, 25(4), 401-426.

Smith, P. R. \& Pollard, D. (1986). The role of computer simulations in engineering education. Computer Education, 10(3), 335-340.

Smith, S. (2003, September). Vital virtual hands-on learning. Techniques, 35-37.

Young, H. P. (2009). Learning by trial and error. Games and Economic Behavior, 65(2), 626643.

Zacharia, Z. C. (2007). Comparing and combining real and virtual experimentation: an effort to enhance students' conceptual understanding of electric circuits. Journal of Computer Assisted Learning, 23(2), 120-132. 